Ann. Biol. anim. Bioch. Biophys., I975, 15 (3), 525-528.

\title{
EFFET DU « LAVAGE » SUR LA CONSERVATION DES SPERMATOZOÏDES DE BOUC A BASSE TEMPÉRATURE
}

\author{
J. M. CORTEEL \\ avec la collaboration technique de G. BARIL \\ Station de Recherches sur la Physiologie de la Reproduction, \\ Centre de Recherches de Tours, I. N. R. A., \\ Nouzilly, 37380 Monnaie, B.P. 1 \\ RÉSUMÉ
}

Dans une proportion non négligeable d'éjaculats de bouc congelés, le pourcentage de spermatozoïdes réanimés au dégel diminue avec le temps de conservation dans l'azote liquide. Cette diminution a été estimée à $\mathrm{I} 6,3$ p. Ioo et 22 p. Ioo pour des durées de conservation de 3-9o jours et de 9I-I 80 jours. Lorsqu'on lave les spermatozoïdes avant de les diluer et de les congeler, cette diminution est annulée pour les conservations de 3-9o jours et réduite à I, 3 p. Ioo seulement pour celles de $9 \mathrm{I}-\mathrm{I} 80$ jours.

\section{IN'TRODUCTION}

La congélation du sperme de bouc a déjà été réalisée et la fécondation obtenue avec les spermatozoïdes dégelés et réanimés (WAIDE et NIWA, I96I ; BONFERT, I965 ; HAHN, I969 ; CoRTEEL et al., I972). Cependant, la proportion des éjaculats qui donnent de bons résultats de fécondation après congélation est faible et dans certains d'entre eux le pourcentage de spermatozoïdes réanimés au dégel diminue avec le temps de conservation dans l'azote liquide. Cette proportion a pu être améliorée en lavant et centrifugeant les spermatozoïdes avant de les diluer dans un milieu de conservation adapté (CORTEEL, I974, I975). Dans ce travail, nous avons cherché à savoir si l'effet bénéfique du lavage des spermatozoïdes portait aussi sur leur conservation à l'état congelé. 


\title{
MATÉRIEL, ET MÉTHODES
}

\author{
I. - Éjaculats dilués sans lavage préalable
}

Immédiatement après la récolte, les éjaculats sont dilués à $30^{\circ} \mathrm{C}$ dans du lait de vache écrémé reconstitué contenant $0,05 \mathrm{M}$ de glucose. La dilution est telle que chaque ml de scmence contient $I \times I 0^{9}$ spermatozoïdes. Les éjaculats sont alors refroidis à $+4^{\circ} \mathrm{C}$ puis glycérolés par l'adjonction d'un volume égal du même dilueur contenant en plus I 4 p. Ioo de glycérol. Après trois heures d'équilibration, la semence est répartie dans des paillettes de $0,2 \mathrm{ml}$ et congeléc dans les vapeurs d'azote. Dégelée 24 à $4^{8}$ heures plus tard à $37^{\circ} \mathrm{C}$, elle est rediluée aur/5 dans le dilueur de congélation de façon à réduire la concentration à $100 \times 10^{6} \mathrm{spz} / \mathrm{ml}$. Ellle est examinée au microscope à contraste de phase pour estimation immédiate du pourcentage de spermatozoïdes mobiles. Cet examen est répété après des temps de conservation variant de 3 à 180 jours.

\section{2. - Éjaculats lavés}

Dès la récolte, les éjaculats sont lavés deux fois dans une solution de Krebs-Ringer-phosphate-glucose et centrifugés à $20^{\circ} \mathrm{C}$ et $700 \mathrm{~g}$; le surnageant est éliminé. Au terme de la seconde centrifugation, les spermatozoïdes sont dilués à la même concentration et traités de la même manière que ceux des éjaculats non lavés.

\section{3. - Expression des résultats}

La diminution du taux de spermatozoïdes mobiles au cours de la conservation est exprimée en pour cent par rapport au taux de spermatozoïdes mobiles observé 24 à 48 heures après la congélation considéré comme le taux initial de réviviscence. La proportion d'éjaculats inutilisables est calculée sur ceux dont le pourcentage de spermatozoïdes estimés mobiles au dégel devient inférieur à 33 p. Ioo.

\section{RÉSUL'TA'TS}

N'ont été conservés pour l'expérience que les éjaculats qui présentaient de 33 à 66 p. Ioo de spermatozoïdes mobiles 24 à 48 heures après congélation soit 5 I et 27 éjaculats avec des moyennes respectives de 44,4 et 43,7 p. Ioo pour les éjaculats non lavés et lavés.

La diminution du taux de spermatozoïdes mobiles pendant la conservation est indiquée au tableau $I$ et la proportion d'éjaculats qui deviennent inutilisables au tablean 2.

\section{TABI,EAU I}

Diminution (en p. Ioo) du taux initial de spermatoziodes mobiles 'n fonction de la duré de conservation de la semence et de la technique de congélation ( ) : nombre d'éjaculats examinés

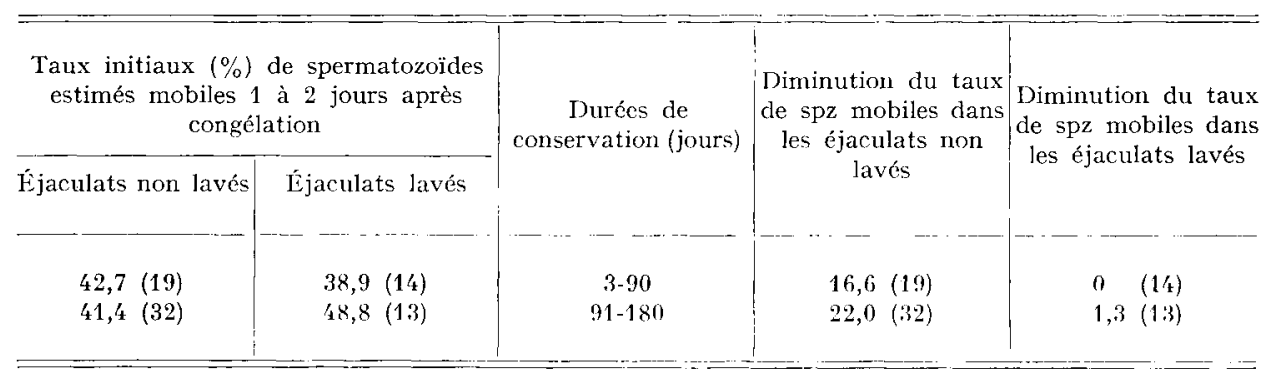


I a diminution du pourcentage de spermatozoïdes mobiles dans les éjaculats lavés est nulle pour des durées de conservation n'excédant pas trois mois et de $\mathrm{I}, 3$ p. Ioo seulement pour des durées comprises entre 3 et 6 mois. Dans les éjaculats non lavés, pour des durées de conservation équivalentes les diminutions sont respectivement de 16,6 et 22 p. Ioo.

TABLEAU 2

Augmentation (en p. roo) des éjaculats inutilisables avec la durée de conservation et la technique de congélation ( ) : nombre d'éjaculats examinés

\begin{tabular}{|c|c|c|}
\hline $\begin{array}{c}\text { Durée de conservation } \\
\text { (jours) }\end{array}$ & Éjaculats non lavés & Ejaculats lavés \\
\hline $\begin{array}{c}3-90 \\
91-180\end{array}$ & $\begin{array}{ll}10,7 & (19) \\
31,3 & (32)\end{array}$ & $\begin{array}{lr}0 & (1 / 4) \\
7,7 & (13)\end{array}$ \\
\hline
\end{tabular}

Ies taux d'éjaculats devenant inutilisables sont de o p. Ioo dans les éjaculats lavés contre I0,7 p. Ioo dans les éjaculats non lavés pour des conservations de 3-90 jours et de 7,7 vs $3 \mathrm{I}, 3$ p. Ioo pour des durées de conservation de $9 \mathrm{I}-\mathrm{I} 80$ jours. Ire seul éjaculat lavé devenu inutilisable présentait 33 p. Ioo seulement de spermatozoïdes mobiles 24-48 heures après congélation.

\section{DISCUSSION ET CONCLUSION}

La diminution du taux de spermatozoïdes mobiles pendant la conservation à basse température a été observée dans la semence de taureau mais après des temps de conservation de plusieurs années (MiXner et Wiggin, Ig64; MiXnER, I968). Le déclin de la fécondance de ces spermatozoïdes interviendrait dès le début de la seconde année de conservation pour des causes non encore élucidées (SALISBury et HART, I970). Chez le bélier, SALAMON (I972) et SALAMON et VISSER (I974) n'observent pas de phénomènes semblables pour des durées de conservation de 3 et 5 ans.

I a diminution du taux de spermatozoïdes mobiles que nous avons observée intervient dès les trois premiers mois de conservation. La rapidité avec laquelle elle se produit paraît caractéristique du sperme de bouc. Elle semble devoir être reliée à une action délétère d'un composant du plasma séminal sur le devenir des spermatozoïdes congelés puisqu'en l'absence de plasma, il n'y a plus de diminution pour les durées de conservation étudiées.

On sait depuis longtemps que le plasma séminal n'est pas le meilleur milieu de conservation des spermatozoïdes. La consommation de glucose $\mathrm{C}_{14}$ par les spermatozoïdes de taureau est réduite lorsque ceux-ci sont conservés avec leur plasma séminal (FLIPSE, I954). Il est peu probable que le métabolisme du spermatozoïde soit perturbé à la température de $-\operatorname{Ig} 6^{\circ} \mathrm{C}$. Par contre, $1^{\prime}$ inaptitude du spermatozoïde de bouc à se conserver à basse température pourrait résulter d'une inhibition métabolique impor- 
tante survenue au cours des manipulations du sperme avant de le congeler comme le suggèrent les résultats antérieurs (COR'TEEL, I974). Quoi qu'il en soit, dans nos conditions expérimentales, le lavage améliore la conservation des spermatozoïdes de bouc dans l'azote liquide.

Rę̧u pour publication en avril 1975.

\title{
SUMMARY
}

\author{
FFFECT OF WASHING ON DEEP FROZEN GOAT SEMEN PRESERVATION
}

In deep-frozen-thawed goat semen, the percentage of mobile sperm decreases by i6,3 p. I00 and $22,0 \mathrm{p}$. Ioo in the three and six month periods following deep-freezing. Washing the spermatozoa prior to freezing reduces the decrease down to o p. Ioo and $\mathrm{I}, 3 \mathrm{p}$. Ioo in the same periods.

\section{RÉFÉRENCES BIBLIOGRAPHIQUES}

Bonfert A., r965. Possibilités et limites de l'insémination artificielle caprine. Tierzuchter, 17, I54-I56.

Corteel J. M., Courot M., Ortavant R., r972. Fertility of multiparous goats inseminated with liquid or deep frozen semen after hormonal synchronization of ostrus before the onset and in the course of the Breeding Season. VIIth Intern. Congr. Anim. Reprod. Artif. Insem. Munich, 2, roog-Ior4.

Corteel J. M., I974. Viabilité des spermatozoïdes de bouc conservés et congelés avec ou sans leur plasma séminal : effet du glucose. Ann. Biol. anim. Bioch. Biophys., 14, 741-745.

Corteel J. M., I975. The use of progestogens to control the cestrus cycle of the dairy goat. Ann. Biol. anim. Bioch. Biophys. (Sous presse).

FLIPSE R. J., I954. Metabolism of bovine semen. I. Uptake of gllicose $C_{14}$ by bovine spermatozoa. J. Dairy Sci., 37, 425-430.

Mixner J. P., Wiggin, I964. The effects of ageing on the motility and fertility of frozen bull semen. Proc. Vth Intern. Congr. Anim. Reprod. Artif. Insem. Trento, 3, $264-268$.

Mixner J. P., I968. Fertility of bull semen frozen for twelve years. Proceed. VIth Intern. Congr. Anim. Reprod. Artif. Insem. Paris, 1095-1098.

Salamon S., I972. Fertility of ram spermatozoa frozen-stored for three years. Proceed VIIth Intern. Congr. Anim. Reprod. Artif. Insem. Munich, 2, 1493 -I 495.

Salamon S., Visser D., I974. Fertility of ram spermatozoa frozen stored for 5 years. J. Reprod. Fert., 37, $433-435$.

Salisbury G. W., Hart R. G., I970. Gamete aging and its consequences. Biol. Reprod. Suppl., 2, I-I3. 